Abstract 585 Table 1 Comparison of the cases according to the learning curve and improvement of the technique

\begin{tabular}{llll}
\hline & First 10 patients & Last 8 patients & $\mathbf{p}$ \\
\hline AGE & $64,50 \pm 11,655$ & $64,25 \pm 9,94$ & $>0,05$ \\
BMI & $27,268 \pm 3,32$ & $27,20 \pm 4,57$ & $>0,05$ \\
N $^{\circ}$ SN MAPEADOS & $1(0-3)$ & $2(1-3)$ & 0,006 \\
N $^{\text {SN FALLADOS }}$ & $1(0-2)$ & $0(0-1)$ & 0,003 \\
N $^{\circ}$ TOTAL SN & $17(1-42)$ & $21(1-44)$ & $>0,05$ \\
REMOVE & & & \\
\hline
\end{tabular}

BMI: Body mass index $\left(\mathrm{kg} / \mathrm{m}^{2}\right)$, SN: Sentinel Node.

lymphadenectomy between June 2019 and March 2020 at the Fundación Jiménez Díaz University Hospital.

We used indocyanine green powder for injection, with a concentration of 25 milligrams (mg). We proceeded to dissolve it in 10 cubic centimeters (cc) of distilled water to avoid precipitation of the marker; obtaining a final concentration of $2.5 \mathrm{mg}$. After which, we injected $2 \mathrm{cc}$ of the prepared solution into the cervix at the 3 and 9 o'clock positions at a depth of $1 \mathrm{~cm}$ through Abbocath $\mathrm{N}^{\circ} 12 \mathrm{G}$ (figure 1).

Results Eighteen patients were included, analyzing a total of 26 sentinel nodes: 24 pelvic and 2 paraortic; and a total of 273 lymph nodes (sentinel and non-sentinel nodes): 83 right pelvic, 86 left pelvic and 104 paraortic. All nodes were negative for metastasis.

Global and bilateral detection rates were $77.78 \%$ and $50 \%$ respectively. The Negative Predictive Value and sensitivity were $100 \%$. No significant difference in morbimortality was found between performing only Sentinel Lymph Node technique or systematic lymphadenectomy; but the association with quality of life was significant, with better results for those who only underwent the sentinel lymph node technique versus systematic lymphadenectomy ( $0 \%$ vs $77 \%$ ).

However, we observed at the beginning of the study bilateral detection was very limited. This could have been due to a failure in the tracer injection technique in our first 10 patients. Since the standardization of the technique we obtained a considerable improvement in bilateral detection; $87.5 \%$ (before technical standardization 20\%). This supports the theory that technique is the most important factor in detection (Rossi, 2019). On the other hand, it is important to assess the learning curve, considered an independent factor that can influence the quality of the technique (table 1).

Conclusion The global and bilateral detection rates of the Sentinel Lymph Node Technique by immunofluorescence were $77.78 \%$ and $50 \%$ respectively, obtaining a Negative Predictive Value and Sensitivity of $100 \%$. Sentinel Node Biopsy is a valid technique to predict lymphatic affectation in early endometrial cancer, with lower morbimortality than systematic lymphadenectomy (figure 2).

Disclosures No conflict of interest to disclose.

\section{EARLY STAGE, LOW GRADE ENDOMETRIAL ADENOCARCINOMA IN REPRODUCTIVE AGED WOMEN. AN EVALUATION OF PATIENT PERSPECTIVES OF DIAGNOSIS, TREATMENT DECISION MAKING, MANAGEMENT, FERTILITY AND SURVIVORSHIP: A QUALITATIVE STUDY}

${ }^{1}$ Alina Roman, ${ }^{2}$ Michelle Peate, ${ }^{3}$ Yasmin Jayasinghe, ${ }^{4}$ Orla M Mcnally. ${ }^{1}$ The Royal Women's Hospital; Gynaecology Oncology, Melbourne Australia; ${ }^{2}$ The Royal Women's Hospital; The University of Melbourne, Melbourne Australia; ${ }^{3}$ The Royal Women's Hospital; The Royal Children's Hospital, Melbourne Australia; ${ }^{4}$ The Royal Women's Hospital; Peter Maccallum Cancer Centre, Melbourne Australia

\subsection{6/ijgc-2020-ESG0.213}

Background Endometrial cancer is rare in reproductive aged women. Standard surgical treatment can impact fertility. Nonstandard conservative management with progestins can be used in very specific populations to give women an opportunity for childbearing. ${ }^{1}$ The treatment experiences of this cohort have not been well studied, and unmet needs not identified or explored. This study aims to explore the experiences of reproductive aged women with early endometrial cancer across

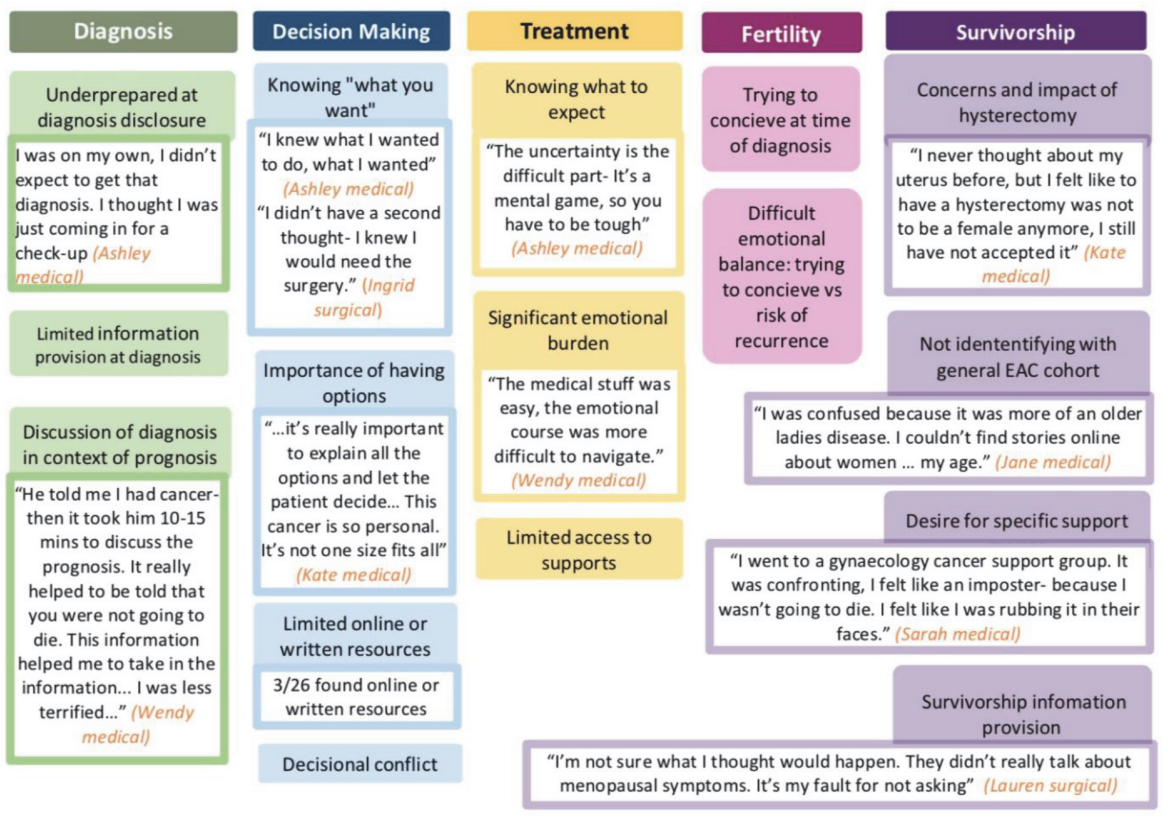

Abstract 589 Figure 1 Common themes identified in the experience of reproductive aged women with early EAC 
diagnosis, decision making, treatment, fertility and survivorship.

Methodology A mixed methods retrospective cohort study was undertaken utilising an online survey and semi-structured interviews addressing themes of diagnosis, management decision making, treatment, fertility and survivorship. Women aged 18-40 years with early EAC, treated at the Royal Women's Hospital (RWH), Melbourne, Australia were identified. Seventy-five women were invited to participate. Online survey links were distributed via mobile text message along with an invitation for interview. Survey responses were collated, and interview transcripts were thematically analyzed using a grounded theory approach.

Results Twenty-six surveys and 14 interviews were completed. Medical management: 20 surveyed 7 interviewed (mean age 33ys) Surgical management: 6 surveyed 7 interviewed (mean age 38 yrs). Responses highlight the shock of the diagnosis and improvements which could be made when communicating the diagnosis. Common themes also included a strong desire for women to be presented with treatment options, suboptimal information provision around treatment options and implications for fertility and long term survivorship as well as the significant emotional burden across all aspects of the treatment journey. Women consistently expressed they did not identify with the wider EAC cohort or other reproductive aged women with cancer and had a strong desire for specific cancer support services. Overall 20 themes across 5 domains were identified. Examples in figure 1.

Conclusion This study examines the experience of young women diagnosed with early EAC. It highlights unmet needs, particularly around available supports and provision of information. These needs should be considered in the future management of these cancer patients.

Acknowledgements To the participants for sharing their experiences.

Disclosures No authors have disclosures to report.

\section{REFERENCE}

1. ESMO-ESGO-ESTRO Endometrial Consensus Conference Working Group. ESMOESGO-ESTRO Consensus Conference on Endometrial Cancer Diagnosis, Treatment and Follow-up. Internat J Gynaecol Cancer 26, 2016.

\section{MISMATCH REPAIR PROTEIN EXPRESSION DEFECTS IN ENDOMETRIOID ENDOMETRIAL ADENOCARCINOMA}

${ }^{1}$ Mark Brincat, ${ }^{2}$ Tiffany Buhagiar, ${ }^{2}$ Sharon Falzon, ${ }^{2}$ Sabrina Ariff, ${ }^{2}$ Simona Bugeja, ${ }^{3}$ James Mark Debono, ${ }^{2}$ Yves Muscat Baron, ${ }^{4}$ Neville Calleja. ${ }^{1}$ Royal London Hospital; ${ }^{2}$ Mater Dei Hospital; ${ }^{3}$ Sir Anthony Mamo Oncology Hospital; ${ }^{4}$ Directorate for Health Information and Research

\subsection{6/ijgc-2020-ESGO.214}

Introduction/Background Endometrial cancer has been shown to be the sentinel cancer in over half the female patients with heritable mismatch repair (MMR) mutations as part of Lynch syndrome. Immunohistochemical testing for MMR protein expression in endometrial cancer is the first screening test identifying cases that potentially harbour familial cancer syndrome-related mutations. MMR has also become a biomarker to predict response to targeted therapeutics such as in immune-checkpoint blockade. This is the first study to describe the prevalence of MMR protein expression defects in Maltese endometrial carcinoma patients and the correlation with patient age at diagnosis.
Methodology 200 endometrioid endometrial cancer cases were retrospectively identified from the Mater Dei Hospital laboratory information system and categorized into three arms: the first consisting of 151 cases over the age of 50 at diagnosis, the second consisting of 49 cases at or under the age of 50 at diagnosis and a control group consisting of 30 patients who underwent endometrial tissue sampling for benign conditions. H\&E slides for these cases were re-examined by an independent pathologist to confirm the diagnosis as well as to identify the block best representing the tumour. Four new slides per case were recut and immunohistochemistry performed for MLH1, PMS2, MSH2, and MSH6 MMR proteins. Protein expression was analysed semiquantitatively using Allred score.

Results In the overall cohort $69 \%$ of cases were MMR proficient while $31 \%$ of cases were deficient for one or more MMR proteins. Dual loss of the MLH1 and PMS2 heterodimer protein expression was the most common deficiency and occurred in $24.5 \%$ of the EEC population. Loss of MSH2-MSH6 heterodimer protein expression was less common and represented 3.2\% of MMR-deficient cases. Well differentiated tumours had a $76.5 \%$ proficiency rate as opposed to grade $2 / 3$ disease with $53.2 \%$ and $52.9 \%$ proficiency rate respectively. There was no statistically significant difference in

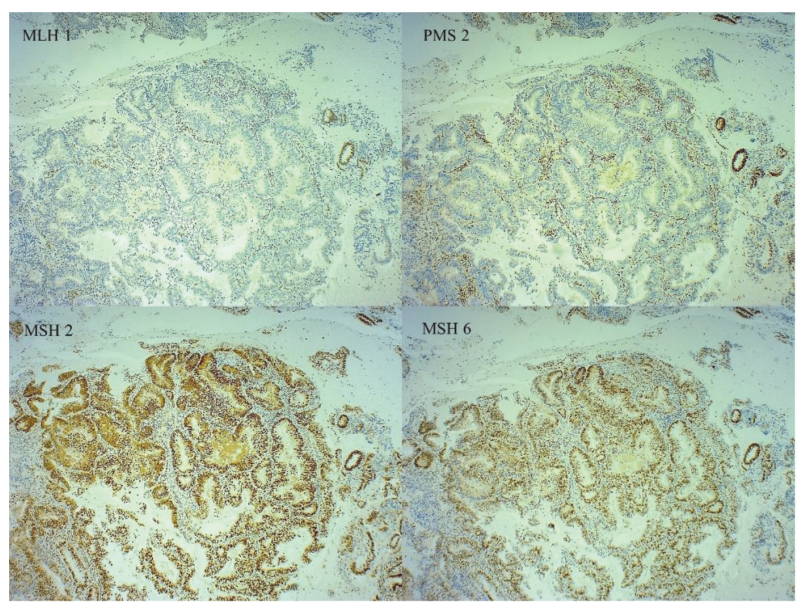

Abstract 597 Figure 1

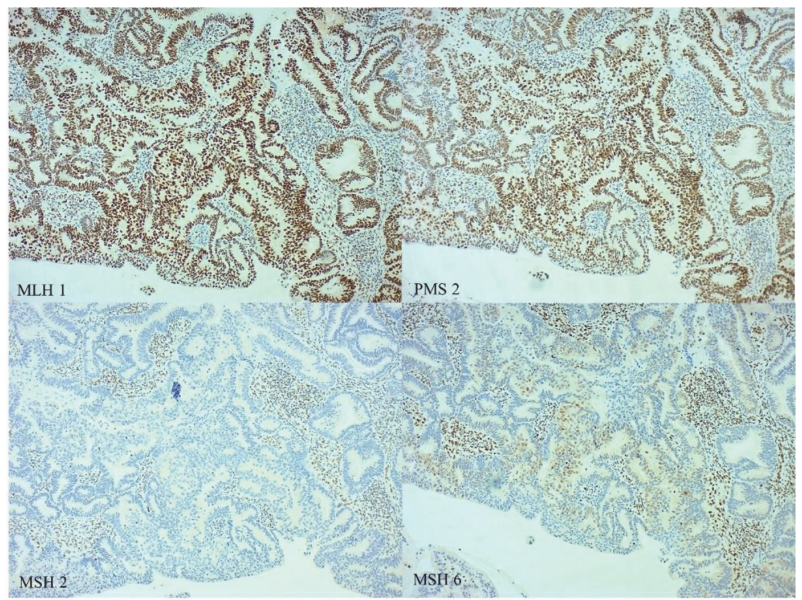

Abstract 597 Figure 2 